\title{
Diarrheal Morbidity During Hematopoietic Cell Transplantation: The Diagnostic Yield of Stool Cultures
}

\author{
Tamar Berger (D) - Odil Giladi · Dafna Yahav · Haim Ben-Zvi • \\ Oren Pasvolsky · Liat Shargian-Alon · Ofir Wolach • Uri Rozovski • \\ Pia Raanani · Moshe Yeshurun
}

Received: December 27, 2020 / Accepted: February 10, 2021 / Published online: March 4, 2021

(C) The Author(s) 2021

\begin{abstract}
Introduction: Diarrhea affects a significant proportion of patients undergoing hematopoietic cell transplantation (HCT). We explored the diagnostic yield of stool cultures for enteric pathogens among patients undergoing HCT.

Methods: This is a single-center, retrospective study. Between 5/2007 and 4/2020, consecutive patients who underwent HCT were included if inpatient bacterial stool cultures were collected. Patient characteristics, results, and timing of stool cultures obtained during hospitalization were collected.
\end{abstract}

T. Berger $(\varangle)$ O. Giladi · O. Pasvolsky ·

L. Shargian-Alon · O. Wolach · U. Rozovski .

P. Raanani · M. Yeshurun

Institute of Hematology, Davidoff Cancer Center,

Rabin Medical Center, Petah Tikva, Israel

e-mail: tamaritayberger@gmail.com

T. Berger · D. Yahav · O. Pasvolsky .

L. Shargian-Alon · O. Wolach · U. Rozovski .

P. Raanani - M. Yeshurun

Sackler Faculty of Medicine, Tel Aviv University, Tel

Aviv, Israel

D. Yahav

Infectious Diseases Unit, Rabin Medical Center,

Beilinson Hospital, Petah Tikva, Israel

H. Ben-Zvi

Microbiology Laboratories, Rabin Medical Center,

Beilinson Hospital, Petah Tikva, Israel
Results: A total of 1072 individuals underwent autologous $(n=603)$ and allogeneic $(n=469)$ HCT. Overall, 947 stool culture samples were obtained from 561 (52\%) patients with diarrheal illness during hospitalization for HCT. Most (99\%) samples were obtained beyond 3 days of admission, mainly (77\%) during neutropenia. Overall, only four $(0.42 \%)$ (autologous, $n=3$; allogeneic, $n=1$ ) patients had a positive stool culture and in all cases Campylobacter spp. were the pathogens identified. The number of stool cultures needed-to-test to diagnose one case of bacterial infection was 237 . The cost of diagnosing one case of bacterial diarrhea was US $\$ 8770$. Patients with a positive stool culture did not have discerning characteristics.

Conclusions: In our experience, the yield of stool cultures for enteropathogens in patients undergoing HCT is extremely low and thus should be avoided in most cases.

Keywords: Diarrheal morbidity; Hematopoietic cell transplantation (HCT); Stool cultures 


\section{Key Summary Points}

Why carry out this study?

Diarrhea is common among patients undergoing hematopoietic cell transplantation (HCT), especially at the time of neutropenia.

Bacterial stool cultures are frequently obtained in the evaluation of diarrheal morbidity in this setting; however, its yield is unclear.

This is the largest study aiming to assess the yield of bacterial stool cultures in the evaluation of diarrheal morbidity during patients' admission for HCT.

\section{What was learned from the study?}

We found high frequency of stool culture sampling, yet a very low $(0.42 \%)$ yield of positive stool cultures obtained from patients with diarrheal illness during hospitalization for HCT.

Stool cultures for enteropathogens in patients undergoing HCT should be avoided.

\section{DIGITAL FEATURES}

This article is published with digital features, including a summary slide, to facilitate understanding of the article. To view digital features for this article go to https://doi.org/10.6084/ m9.figshare.13744363.

\section{INTRODUCTION}

Diarrhea is a frequent complication in patients undergoing autologous and allogeneic hematopoietic cell transplantation (HCT), affecting approximately $50 \%$ of patients [1]. While most commonly attributed to mucosal injury induced by high-dose chemo/radio therapy, multiple other etiologies may be involved. The incidence of infectious gastroenteritis associated with HCT reported in the literature varies from $13 \%$ to $40 \%$ [2-4], mostly due to Clostridioides difficile (CD) or viral infection.

According to the "3-day rule" dogma, diarrhea presenting after a patient has been at the hospital for 3 days should not be submitted for routine stool cultures for enteropathogens since they are unlikely to recover any pathogens [5-7]. While this policy is well established in the general population, its validity in patients with hematological malignancies and specifically in patients undergoing HCT is debatable, as data in these populations are scarce. Some argue that hemato-oncology patients should be exempted from the "3-day rule" and send stool sample for culture at any hospitalization time point, only if they are older than 65 years or while neutropenic. Nevertheless, in the HCT setting, most patients who develop diarrhea, do so around the neutropenic phase.

Few very small non-homogeneous retrospective and prospective studies reported high rates of diarrhea, yet with low yield of positive stool cultures among patients undergoing HCT [8-11].

In the present study, we aimed to assess the diagnostic yield of stool cultures for enteric pathogens other than $\mathrm{CD}$ in the evaluation of diarrheal morbidity in a large cohort of patients admitted for HCT at our institution. We hypothesized that the "3-day rule" may be invalid in this setting. This is the largest study assessing the yield of bacterial stool cultures during patients' admission for HCT.

\section{METHODS}

\section{Study Design}

This is a single-center, retrospective study. Between May 2007 and April 2020, consecutive adult patients who underwent autologous and allogeneic HCT at the bone marrow transplantation unit in the Institute of Hematology at the Rabin Medical Center were included if inpatient bacterial stool cultures were collected. Rabin 
Medical Center includes 1300 inpatient beds, 13 beds of which are devoted to the HCT service. Bacterial stool cultures were obtained only from patients reporting having three or more loose stools per day. Patients were identified using the bone marrow transplantation unit and the microbiology laboratory electronic medical records (EMRs). Clinical data were collected from EMRs.

Data collection included patient demographics, diagnosis, type of HCT performed (autologous or allogeneic), results and timing of stool cultures obtained during hospitalization, laboratory tests, and associated symptoms (i.e., fever, elevated inflammatory markers) in proximity to the time the stool cultures were obtained.

The study was approved by the Rabin Medical Center Institutional Review Board. Informed consent was waived because of the retrospective design of the study. The study was performed in accordance with the Helsinki Declaration of 1964, and its later amendments.

\section{General Antimicrobial and Prevention Measures}

All patients were placed in a protective environment consisting of well-sealed single bedrooms with at least 12 cycles of air exchanges per hour, HEPA filters, directed airflow, consistent positive air pressure, and self-closing doors. Patients received a low microbial diet and were managed under strict contact precaution measures by hospital personnel to reduce nosocomial infections. Upon admission, surveillance cultures were taken, namely, nose swabs for methicillin-resistant Staphylococcus aureus (MRSA), and rectal swabs for vancomycin-resistant enterococci (VRE), and carbapenem-resistant Enterobacteriaceae (CRE).

\section{Antibiotic Strategy}

All transplanted patients were treated with oral antiviral prophylaxis consisting of acyclovir $400 \mathrm{mg}$ BID. Patients undergoing allogeneic
HCT were given oral antibacterial prophylaxis consisting of ciprofloxacin $500 \mathrm{mg}$ BID and during most of the study period also oral antifungal prophylaxis (fluconazole $150 \mathrm{mg}$ QD). During most of the study period, patients undergoing autologous HCT were given antibacterial prophylaxis, consisting of ciprofloxacin $500 \mathrm{mg}$ BID, but without antifungal prophylaxis. Only between October 2012 and July 2016, patients undergoing autologous HCT were not given any antibacterial prophylaxis.

\section{Endpoints and Statistical Analysis}

Primary outcome was the rate of positive bacterial stool cultures obtained during hospitalization for HCT.

Secondary outcomes were rates of positive stool cultures among patient subpopulations (i.e., autologous vs. allogeneic HCT); and at different time points during hospitalization, namely within the first 3 days of admission vs. later time points.

Patient characteristics were summarized using frequencies (number and percentages) for categorical variables and median (range) or mean (SD) for continuous variables, as appropriate. We planned to perform a univariable and multivariable analyses of risk factors for positive stool cultures. These were not conducted because of the low rate of positive cases.

\section{Microbiological Methods}

Stool samples for culture were sent to the microbiology laboratory in aseptic containers. The samples were processed according to accepted procedures [12]. Briefly, stool was plated on SS agar, campylobacter blood plates, and selenite broth. If growth was noticed, enterotest and Chrom agar salmonella were used. If positive, agglutination tests were conducted. Growth of either Salmonella spp., Shigella spp., or Campylobacter spp. was considered positive stool culture.

The same methods apply to all transplant centers in Israel. 


\section{RESULTS}

Between May 2007 and April 2020, 1072 individuals underwent HCT (autologous, $n=603$; allogeneic, $n=469)$. Overall, 947 stool cultures were obtained from $561(52 \%)$ patients (autologous, $n=356$; allogeneic, $n=205$ ) with diarrhea during HCT, in search of a bacterial pathogen (Fig. 1). Indications for HCT in these patients are presented in Table 1 . Rates of stool culture sampling were higher among patients undergoing autologous HCT compared to patients undergoing allogeneic HCT (59\% vs. $44 \%, P<0.001)$. The number of stool samples per patient varied: one culture was collected from 327 (58\%) patients and two or more cultures from $234(42 \%)$ patients [median 1 (range 1-8)] (Table 2). Importantly, 99\% of the cultures were obtained beyond 3 days of admission and $77 \%(n=727)$ during neutropenia. The median times from the day of admission and the day of HCT to the first stool culture collection were 18 (range 0-64) days and 10 (range - 12 to 61) days, respectively. Over a 13-year period, only 4
$(4 / 947,0.42 \%)$ stool cultures collected from 4 $(4 / 561,0.7 \%)$ different patients (autologous, $n=3$; allogeneic, $n=1$ ) were positive (Fig. 1); these four patients were treated with ciprofloxacin as antibacterial prophylaxis before HCT and in all cases Campylobacter spp. were the pathogens isolated, always from the first stool culture sampled.

None of the four patients with a positive stool culture had gastrointestinal (GI) symptoms upon admission. Patients' symptoms varied from mild diarrhea to profuse watery diarrhea accompanied by vomiting, abdominal pain, and fever. Two patients were neutropenic at the time of stool sampling and none had bacteremia. Upon identification of Campylobacter, patients were treated with a macrolide (azithromycin, $n=3$; clarithromycin, $n=1$ ).

Characteristics of the patients with a positive stool culture are depicted in Table 3.

Between October 2012 and July 2016, 288 stool samples were obtained from 163 patients who underwent autologous HCT without antibacterial prophylaxis in accordance with

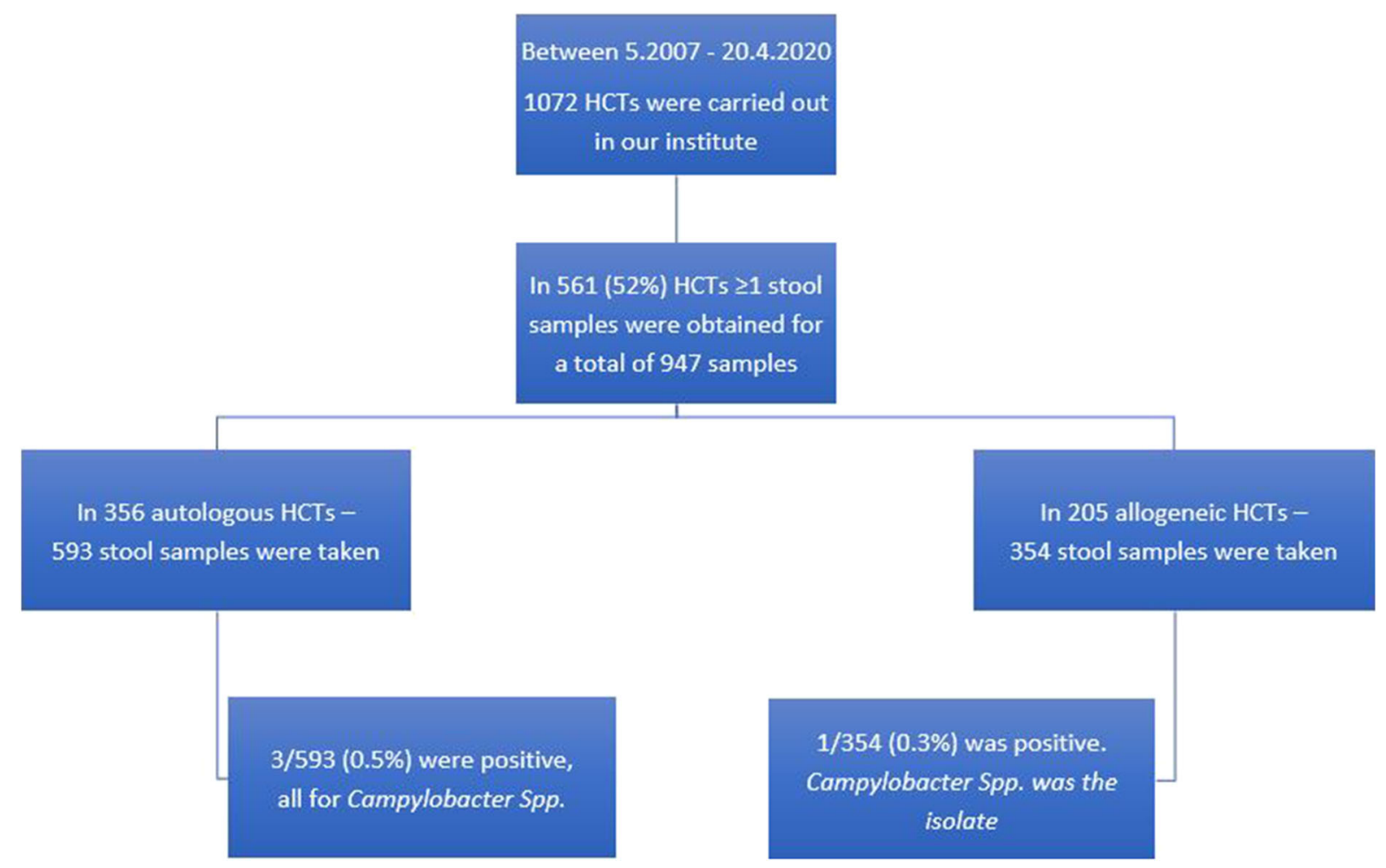

Fig. 1 Study design, positive stool cultures rates according to patients' cohorts. HCT hematopoietic cell transplantation 
Table 1 Indications for hematopoietic cell transplantation (HCT) among patients sampled for stool cultures $(n=561)$

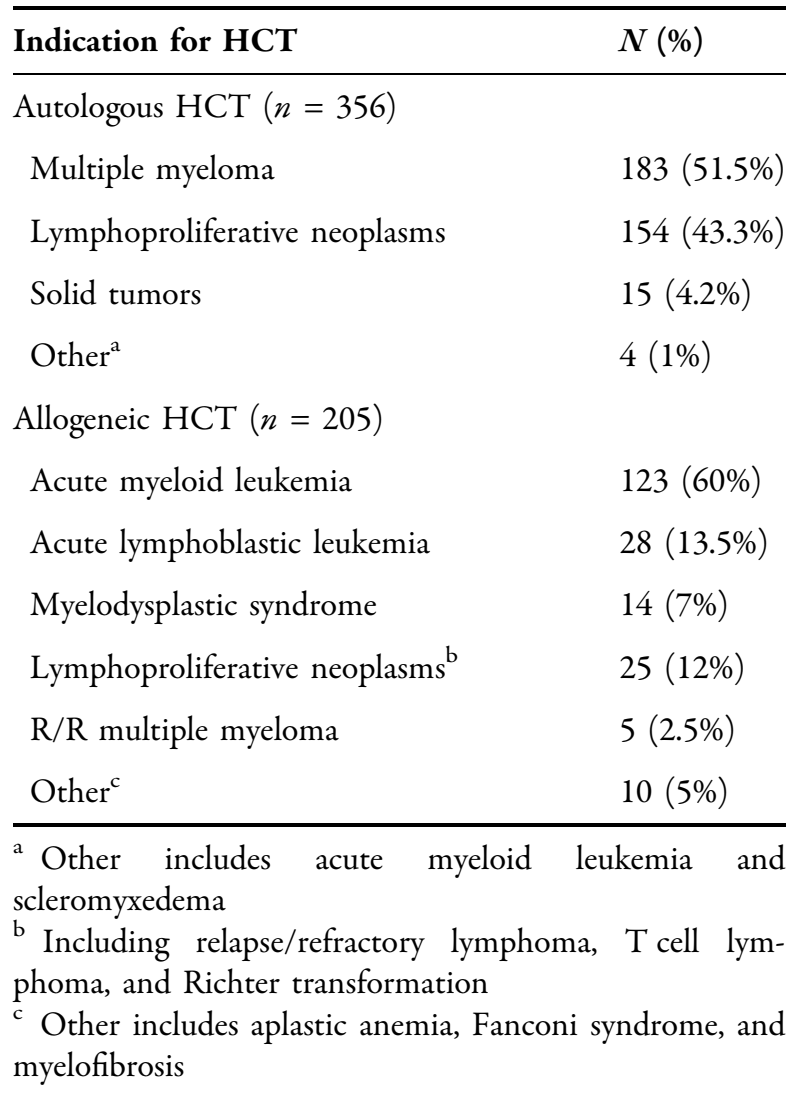

Table 2 Number of stool cultures obtained per patient during admission for hematopoietic cell transplantation

\begin{tabular}{ll}
\hline $\begin{array}{l}\text { Number of stool cultures } \\
\text { per patient }\end{array}$ & $\begin{array}{l}\text { Number of patients (\% of } \\
\text { patients) }\end{array}$ \\
\hline 1 & $327(58 \%)$ \\
2 & $138(24.5 \%)$ \\
3 & $60(11 \%)$ \\
4 & $21(4 \%)$ \\
$\geq 5$ & $15(2.5 \%)$ \\
Total & $561(100 \%)$ \\
\hline
\end{tabular}

departmental policy during this period. All 288 stool samples were negative for an enteric pathogen. Overall, the number of stools- needed-to-test to diagnose one case of bacterial (other than CD) infectious diarrhea was 237.

The customary cost of a stool culture, according to the Israel Ministry of Health is approximately 37US \$ per stool culture. Thus, the cost of diagnosing one case of bacterial (non-CD) infectious diarrhea was 8770US \$. These costs do not include the cost and workload of laboratory staff, as well as the inconvenience of stool handling for both patients and staff.

Molecular multiplex stool tests were rarely done at our institute because of high costs. Only two stool samples were analyzed by PCR at later years of the study period. Of those, one was positive, yet the test results did not impact patient management.

\section{DISCUSSION}

Over half of the patients hospitalized for HCT at our institution developed diarrhea and submitted stool specimens for culture. Nevertheless, only 4 out of 947 bacterial stool cultures were positive. The total yield for entropathogenic stool cultures in our cohort was $0.42 \%(0.5 \%$ and $0.3 \%$ among autologous and allogeneic HCT patients, respectively) with the number of stools-needed-to-test to diagnose one case of bacterial (other than CD) infectious diarrhea of 237. Despite the fact that all isolates were Campylobacter spp., they do not represent a nosocomial outbreak since they were diagnosed in different years. The extremely low number of subjects with positive stool cultures in our cohort and lack of specific symptoms did not allow for a comparison analysis between patients with positive and negative stool cultures.

Our results support previous findings of high rates $(40-50 \%)$ of diarrheal morbidity [1], yet with very low yield of bacterial (non-CD) stool cultures among patients undergoing HCT, as shown in Table 4 [8-11].

There are no explicit guidelines for the diagnostic evaluation of diarrhea in patients undergoing HCT outside the general guidelines for oncological patients. The 2017 guidelines of the Infectious Diseases Working Party (AGIHO) 


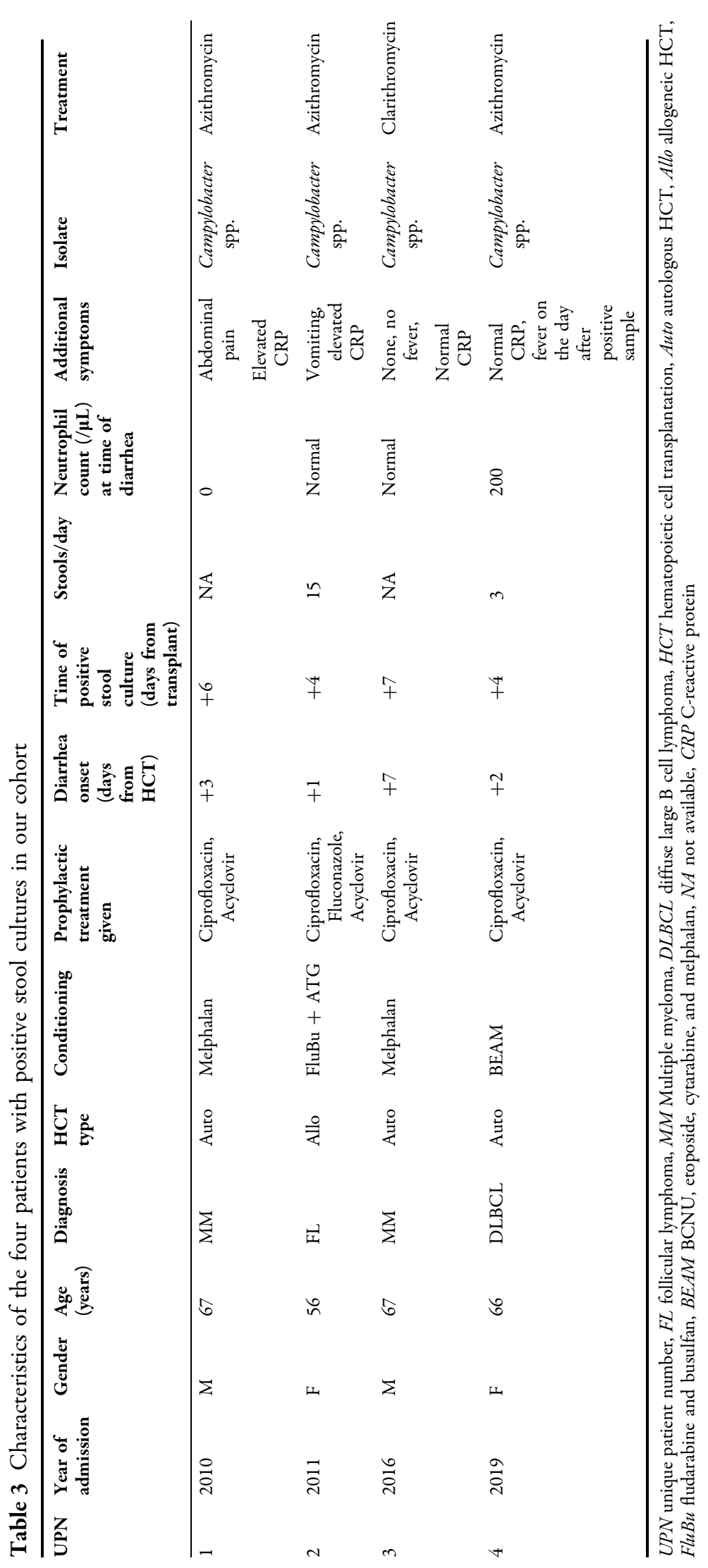


Table 4 Studies evaluating stool cultures yield in hospitalized patients with hematological malignancies, including patients post HCT

\begin{tabular}{|c|c|c|c|c|c|}
\hline Reference & Study population & $\begin{array}{l}\text { Study } \\
\text { design }\end{array}$ & $\begin{array}{l}\text { Total number of } \\
\text { stool cultures }\end{array}$ & $\begin{array}{l}\text { Number of } \\
\text { positive } \\
\text { stool } \\
\text { cultures }\end{array}$ & $\begin{array}{l}\text { Stool } \\
\text { culture's } \\
\text { yield }(\%)\end{array}$ \\
\hline $\begin{array}{l}\text { Smarkos } \\
\text { et al. [8] }\end{array}$ & $\begin{array}{l}\text { Inpatients with different hematological } \\
\text { malignancies }^{\mathrm{a}}\end{array}$ & Retrospective & 217 & 1 & 0.46 \\
\hline $\begin{array}{l}\text { Kamboj } \\
\text { et al. [9] }\end{array}$ & 169 adult and pediatric inpatients post HCT & Retrospective & 345 & 0 & 0 \\
\hline $\begin{array}{l}\text { Van Kraaij } \\
\text { et al. } \\
{[10]}\end{array}$ & $\begin{array}{l}48 \text { adults undergoing } \mathrm{HCT} \text {-samples taken } \\
\text { prior to HCT, at fixed days post transplant } \\
\text { and at the onset of diarrhea and twice } \\
\text { weekly for } 2 \text { weeks thereafter }\end{array}$ & Prospective & $\begin{array}{l}\text { NA } \\
\text { Stool tests taken } \\
\text { in } 36 \text { acute } \\
\text { diarrheal } \\
\text { episodes }\end{array}$ & 0 & 0 \\
\hline $\begin{array}{l}\text { Alejo- } \\
\text { Cancho } \\
\text { et al. } \\
\text { [11] }\end{array}$ & $\begin{array}{l}\text { Adult hematologic patients including } \\
\text { inpatients after cytotoxic agents or auto- } \\
\text { HCT or during the first year after allo- } \\
\text { HCT with acute diarrhea }{ }^{\text {b }}\end{array}$ & Prospective & 95 & 6 & 6.3 \\
\hline $\begin{array}{l}\text { Berger } \\
\text { et al. }\end{array}$ & Adult patients hospitalized for HCT & Retrospective & 947 & 4 & 0.42 \\
\hline
\end{tabular}

$H C T$ hematopoietic cell transplantation, auto-HCT autologous hematopoietic transplantation, allo-HCT allogeneic hematopoietic cell transplantation, $N A$ not available

${ }^{a}$ No mention if patients underwent HCT

b Included heterogenic population of hematological patients

of the German Society of Hematology and Medical Oncology (DGHO) state that infectionrelated diarrhea due to enteric pathogens is a rare event $(0-2.8 \%)$ among patients with cancer in general, advocating testing for these pathogens only in fecal samples taken within $72 \mathrm{~h}$ of hospital admission from symptomatic patients [7], by that adopting the "3-day rule" policy $[5,6]$. On the basis of our local data, $77 \%$ of the patients would have been exempted from the "3-day rule" as they were neutropenic at the time of diarrhea, yet without improving the yield of stool culture sampling. We advocate not to repeat stool culture testing at the same diarrheal episode, since in our experience, all positive cultures were already identified in the initial stool culture performed. This is in line with a prior study demonstrating no excess yield with repetitive stool culture testing of the same patient with diarrheal episode during HCT admission [9].

Campylobacter is a leading cause of foodborne gastroenteritis worldwide [13]. It was not surprising to find Campylobacter spp. as the main-and in our cohort the only-enteric pathogen isolated from stool specimens, since the majority of our patients (including the four patients with positive stool samples) were treated with ciprofloxacin prophylaxis during HCT [14] and campylobacter is notorious for its high rate of resistance to ciprofloxacin in Israel, as well as in other parts of the world [13]. 
Although hematological malignancies and immunosuppression are predisposing factors for campylobacter bacteremia $[15,16]$, none of our patients developed this complication. Of note, trials delineating campylobacter bacteremia in immunosuppressed patients found prognosis to be generally favorable regardless of appropriateness of antibiotic therapy [15, 16]. Boyle et al. reported 12 positive bacterial stool cultures out of 4069 collected among HCT recipients within 1 year of transplantation [17]. None of the patients developed shock or was transferred to an intensive care unit and no attributable mortality was reported.

In contrast to infectious diarrhea due to enteropathogenic bacteria, Clostridioides difficile infection (CDI) is 10 times more commonly diagnosed among HCT patients with diarrheal illness $[9,18]$. In two small prospective studies, diarrhea was very frequent, evident in over half of HCT recipients, with CDI identified in approximately $5 \%$ of patients $[10,19]$. We chose not to assess its frequency in the present study, since data regarding CDI among HCT patients is more solid.

The current study has several limitations. Firstly, this is a retrospective, single-center study. Secondly, usage of quinolone prophylaxis in most of our patients may limit generalization of our results. Lastly, the low number of positive stool cultures in our cohort precluded us from comparing characteristics of patients with positive and negative stool cultures in order to illuminate characteristics that may increase the yield of stool culture testing. However, to the best of our knowledge, and as portrayed in Table 4, this is the largest study assessing the yield of bacterial stool cultures during patients' admission for HCT.

In addition, as applies to all transplant centers in Israel, stool samples were not tested by PCR assays for GI pathogens. It has been suggested that the "3 day rule" does not apply when using PCR of stool samples; however, this stemmed mainly from the test's ability to identify non-bacterial pathogens [20]. Rogers et al. replaced conventional stool testing with a multiplexed PCR assay in HCT recipients. They identified infectious etiologies of diarrhea in a higher proportion of cases; however, they were unable to evaluate the impact of the GI PCR assay on overall antibiotic utilization or time to initiation of appropriate therapy for a diarrheal infection since many patients received antibiotics for reasons other than the treatment of infectious diarrhea. Most common pathogens identified were norovirus and Enteropathogenic Escherichia coli (EPEC) [21]. Since EPEC may be part of an asymptomatic carriage state and its pathogenic role is unclear [21], it seems that identification of viral pathogens or EPEC is irrelevant.

Indeed, we had sporadic stool samples tested by PCR assay with no management impact on patients' care. Thus we believe that although PCR stool testing is more sensitive than bacterial stool cultures in identifying GI pathogens, in most instances, stool PCR and stool cultures obtained from patients beyond 3 days of admission will be of low value and will not change patients' treatment.

\section{CONCLUSIONS}

In this large single-center retrospective study, we found high frequency, yet a very low $(0.42 \%)$ yield of positive stool cultures obtained from patients with diarrheal illness during hospitalization for HCT. This translates into an exaggerated usage of resources, high costs, significant laboratory workload, and high number of specimens needed to test in order to identify one positive stool culture. On the basis of these results, we suggest that the vast majority of patients undergoing HCT should not be exempted from the "3-day rule", and thus should not submit stool specimens for enteropathogenic bacteria (other than CD). Exceptional cases may include patients with persistent diarrhea despite neutrophil recovery, mucositis resolution and negative CDI test, or in cases of local outbreaks. 


\section{ACKNOWLEDGEMENTS}

Funding. No funding or sponsorship was received for this study or publication of this article.

Authorship. All named authors meet the International Committee of Medical Journal Editors (ICMJE) criteria for authorship for this article, take responsibility for the integrity of the work as a whole, and have given their approval for this version to be published.

Prior Presentation. This study was presented as an ASH poster presentation in the 62nd American Society of Hematology (ASH) virtual meeting held on December 2020.

Disclosures. Tamar Berger, Odil Giladi, Dafna Yahav, Haim Ben-Zvi, Oren Pasvolsky, Liat Shargian-Alon, Ofir Wolach, Uri Rozovski, Pia Raanani and Moshe Yeshurun have nothing to disclose.

Compliance with Ethics Guidelines. The study was approved by the Rabin Medical Center Institutional Review Board. Informed consent was waived because of the retrospective design of the study. The study was performed in accordance with the Helsinki Declaration of 1964, and its later amendments.

Data Availability. The datasets generated and/or analyzed during the current study are available from the corresponding author on reasonable request.

Open Access. This article is licensed under a Creative Commons Attribution-NonCommercial 4.0 International License, which permits any non-commercial use, sharing, adaptation, distribution and reproduction in any medium or format, as long as you give appropriate credit to the original author(s) and the source, provide a link to the Creative Commons licence, and indicate if changes were made. The images or other third party material in this article are included in the article's Creative Commons licence, unless indicated otherwise in a credit line to the material. If material is not included in the article's Creative Commons licence and your intended use is not permitted by statutory regulation or exceeds the permitted use, you will need to obtain permission directly from the copyright holder. To view a copy of this licence, visit http://creativecommons.org/licenses/by$\mathrm{nc} / 4.0 /$.

\section{REFERENCES}

1. Hamdeh S, Almonem MA, Elsallabi O. Clinical approach to diarrheal disorders in allogeneic hematopoietic stem cell transplant recipients. World J. 2016;1:12.

2. Yolken RH, Bishop CA, Townsend TR, et al. Infectious gastroenteritis in bone-marrow-transplant recipients. N Engl J Med. 1982;306(17):1010-2.

3. Cox GJ, Matsui SM, Lo RS, et al. Etiology and outcome of diarrhea after marrow transplantation: a prospective study. Gastroenterology. 1994;107(5): 1398-407.

4. Troussard X, Bauduer F, Gallet E, et al. Virus recovery from stools of patients undergoing bone marrow transplantation. Bone Marrow Transplant. 1993;12(6):573-6.

5. Bauer TM, Lalvani A, Fehrenbach J, , et al. Derivation and validation of guidelines for stool cultures for enteropathogenic bacteria other than Clostridium difficile in hospitalized adults. JAMA. 2001;285(3):313-9.

6. Le Guern R, Loïez C, Grandbastien B, Courcol R, Wallet F. Performance of stool cultures before and after a 3-day hospitalization: fewer cultures, better for patients and for money. Diagn Microbiol Infect Dis. $2013 ; 77(1): 5-7$.

7. Schmidt-Hieber M, Bierwirth J, Buchheidt D, et al. Diagnosis and management of gastrointestinal complications in adult cancer patients: 2017 updated evidence-based guidelines of the Infectious Diseases Working Party (AGIHO) of the German Society of Hematology and Medical Oncology (DGHO). Ann Hematol. 2018;97(1):31-49.

8. Samarkos M, Kampouropoulou O, Theofanis V, et al. The 3-day rule for stool cultures: should all patients with haematological malignancies be excluded? Clin Microbiol Infect. 2018;24(12):1342. e1-1342.e3. 
9. Kamboj M, Mihu CN, Sepkowitz K, Kernan NA, Papanicolaou GA. Work-up for infectious diarrhea after allogeneic hematopoietic stem cell transplantation: single specimen testing results in cost savings without compromising diagnostic yield. Transpl Infect Dis. 2007;9(4):265-9.

10. van Kraaij MG, Dekker AW, Verdonck LF, et al. Infectious gastro-enteritis: an uncommon cause of diarrhoea in adult allogeneic and autologous stem cell transplant recipients. Bone Marrow Transplant. 2000;26(3):299-303.

11. Alejo-Cancho I, Fernández Avilés F, et al. Evaluation of a multiplex panel for the diagnosis of acute infectious diarrhea in immunocompromised hematologic patients. PLoS One. 2017;12(11): e0187458.

12. Leber AL, editor. Clinical microbiology procedures handbook. 4th edn. Washington, DC: American Society of Microbiology; 2016.

13. Azrad M, Tkhawkho L, Isakovich N, Nitzan O, Peretz A. Antimicrobial susceptibility of Campylobacter jejuni and Campylobacter coli: comparison between Etest and a broth dilution method. Ann Clin Microbiol Antimicrob. 2018;17(1):23.

14. Yeshurun M, Vaxman I, Shargian L, et al. Antibacterial prophylaxis with ciprofloxacin for patients with multiple myeloma and lymphoma undergoing autologous haematopoietic cell transplantation: a quasi-experimental single-centre before-after study. Clin Microbiol Infect. 2018;24(7):749-54.
15. Hussein K, Raz-Pasteur A, Shachor-Meyouhas Y, et al. Campylobacter bacteraemia: 16 years of experience in a single centre. Infect Dis (Lond). 2016;48(11-12):796-9.

16. O'Hara GA, Fitchett JRA, Klein JL. Campylobacter bacteremia in London: a 44-year single-center study. Diagn Microbiol Infect Dis. 2017;89(1): $67-71$.

17. Boyle NM, Podczervinski S, Jordan K, et al. Bacterial foodborne infections after hematopoietic cell transplantation. Biol Blood Marrow Transplant. 2014;20(11):1856-61.

18. Alonso CD, Treadway SB, Hanna DB, et al. Epidemiology and outcomes of Clostridium difficile infections in hematopoietic stem cell transplant recipients. Clin Infect Dis. 2012;54(8):1053-63.

19. Avery R, Pohlman B, Adal K, et al. High prevalence of diarrhea but infrequency of documented Clostridium difficile in autologous peripheral blood progenitor cell transplant recipients. Bone Marrow Transplant. 2000;25(1):67-9.

20. Beal SG, Velez L, Tremblay EE, Toffel S, Rand KH. The "3-day rule" for stool tests may not apply when using PCR panels. J Clin Microbiol. 2018;56(4):1.

21. Rogers WS, Westblade LF, Soave R, et al. Impact of a multiplexed PCR panel on identifying diarrheal pathogens in hematopoietic cell transplant recipients. Clin Infect Dis. 2020;;71(7):1693-1700. 